\title{
ApUntes aCERCA de la natURALEZA Y LAS vías de COMUNICACIÓn DE COSTA RICA, UNA MIRADA A PARTIR DE UNA EXCURSIÓN DE 1907
}

\footnotetext{
Javier Agüero García.

Egresado del Programa Latinoamericano de Doctorado en Educación, UCR. Magister Scientiae en Historia, UCR y egresado de la Licenciatura en Docencia de la UNED. Excoordinador de la Sección de Historia y Geografía de la Sede de Occidente. Profesor de la Sección de Historia de la Cultura de la Escuela de Estudios Generales de la Sede Rodrigo Facio y de la Sección de Historia y Geografía del Departamento de Ciencias Sociales de la Sede de Occidente. Autor de trabajos relacionados con la historia de la educación. Coautor de artículos acerca de la enseñanza de los Estudios Sociales, e historia de la reproducción de los oficios rurales. Ha impartido los cursos de Historia de la Cultura en las opciones regular y seminario participativo, Historia de las Instituciones de Costa Rica, Historia Antigua Universal, Historia Moderna Universal, Historia Contemporánea Universal, Teoría de la Historia Económica, Temas de Historia Económica en Historia Universal y Formación Ciudadana. jav_aguero@hotmail.com
}

\begin{abstract}
Resumen
En el presente artículo se aborda, a partir de un texto escrito publicado en 1907 por los viajeros españoles José Segarra y Joaquín Juliá, la naturaleza y las vías de comunicación junto con el transporte de Costa Rica. El objetivo fundamental consiste en obtener una visión de lo escrito por foráneos acerca de la Costa Rica durante los umbrales del siglo XX. Se concluye en que los excursionistas se maravillan por la exuberancia natural, contemplada en el país en la abundancia de agua y en la riqueza de los bosques que, junto a un clima generoso, ofrecen oportunidades de crecimiento económico; sin embargo, alertan acerca de las condiciones deficitarias que impedirían esa suerte en lo concerniente a los caminos y a los ferrocarriles.
\end{abstract}

Palabras clave: naturaleza, agua, bosques, vías de comunicación, ferrocarriles, Costa Rica.

\section{Abstract}

This article studies, from a written text published in 1907 by Spanish travelers José Segarra and Joaquín Juliá, the nature and means of communication with the transportation of Costa Rica. The fundamental objective is to get a glimpse of what was written by outsiders about Costa Rica during the threshold of the twentieth century. It is concluded that hikers marvel at the natural exuberance, issued in the country in the water abundance and richness of forests, which along with a generous climate offer opportunities for economic growth. However they warn about the poor conditions, which would prevent that fate, with regard to roads and railways.

Key Words: nature, water, forests, roads, railways, Costa Rica.

\section{Introducción}

Los escritos de los viajeros constituyen una fuente histórica invaluable porque, entre otras razones, son testimonios elaborados al calor de lo observado en el momento por los excursionistas; puede entonces que se asemejen, en su finalidad a aquellos documentos redactados por el padre de la historia, Herodoto de Halicarnaso, durante la Grecia clásica. El aporte de estos escritos da para mucho, porque conforman una fuente de información válida para llegar a determinar lo sucedido y además evidencian el prisma mediante el cual observan los viajeros los fenómenos.

Para el caso centroamericano, los testimonios de los viajeros como Robert Glasgow y Wilhelm Marr han sido objeto de estudio de los historiadores, quienes se han centrado en el análisis de aportes para el siglo XIX. En la centuria siguiente 
Imagen 1: Portada del libro "Excursión por América, Costa Rica" de José Segarra y Joaquín Juliá (1907) Fuente: Tomado con fines didácticos de: Segarra, José y Juliá, Joaquín. (1907). Excursión por América, Costa Rica. San José: Imprenta de Avelino Alsina., s.n.p.
Imagen 2: Lugares visitados por José Segarra y Joaquín Juliá durante su excursión en Costa Rica en 1907. Fuente: Tomado con fines didácticos de: Segarra, José y Juliá, Joaquín. (1907). Excursión por América, Costa Rica. San José: Imprenta de Avelino Alsina., s.n.p.

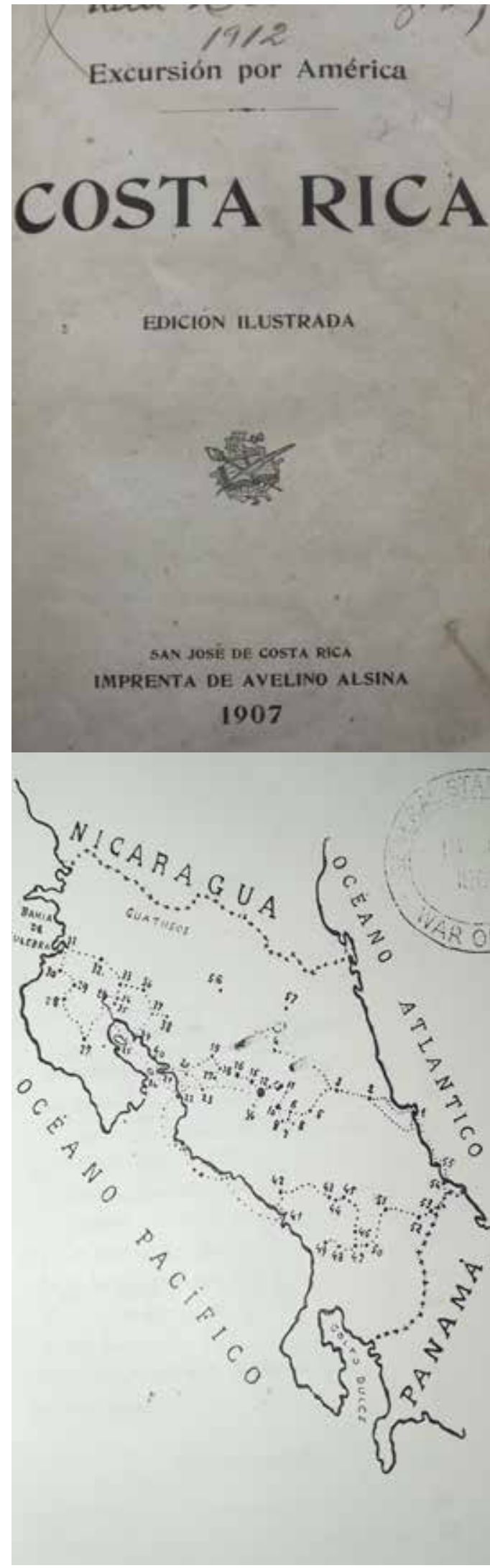

también existen textos de gran valía, como es el caso del estadounidense Dana Munro, que en su condición de becado para realizar estudios doctorales, dejó a la posteridad un excepcional aporte a partir de un ensayo escrito con un marcado énfasis en el rubro de la vida política de los cinco estados del istmo (Molina, 2003).

Para el presente artículo se analiza un texto publicado en 1907 escrito por los excursionistas españoles José Segarra y Joaquín Juliá; autores de otros textos adicionales bajo la serie Excursión por América. Los viajeros llegaron a Limón procedentes de Cuba y prometieron, una vez concluida su aventura en Costa Rica, dirigirse hacia Panamá. La estructura del texto Excursión por América, Costa Rica incluye contenidos acerca de la geografía, la historia, la economía, la política, la literatura y las costumbres; además de incluir descripciones sobre la naturaleza y las vías de comunicación; todos estos temas aparecen mencionados durante un itinerario que abarcó todo lo ancho y lo largo del territorio nacional. Al respecto véase el mapa siguiente.

Los temas analizados en este artículo son la naturaleza y las vías de comunicación. Su elección obedece, en primer lugar, al reciente interés de la historiografía costarricense en abordar temáticas asociadas con el ambiente y los recursos naturales, según se desprende de un balance de los estudios acerca del pasado realizado en 2010 (Clark, Goebel y Rivero, 2014). En tanto que en el caso de las vías de comunicación y de los transportes, conforman tópicos de estudio urgente por parte de quienes se interesan en vincular el desarrollo de los caminos y los medios de locomoción. La historia de las vías de comunicación en Costa Rica está por escribirse aún.

El propósito de este ensayo consiste en analizar cómo observan la naturaleza y las vías de comunicación los viajeros Segarra y Juliá, para así llegar obtener una visión de lo escrito por 
foráneos acerca de la Costa Rica durante los umbrales del siglo XX.

Los autores, además de narrar lo encontrado desde su propia perspectiva a partir de su experiencia personal, también basan su discurso en la consulta de fuentes varias como los textos de personajes de la época colonial, como los del Obispo Pedro Agustín Morel de Santa Cruz, citado por el historiador Ricardo Fernández Guardia. Además hacen referencia directa a los trabajos de Lorenzo Montúfar, Félix Noriega, León Fernández, Joaquín Bernardo Calvo, Francisco Montero, y Francisco María Iglesias, entre otros. También los autores se valen de la valiosa información contenida en las memorias gubernamentales y en el Diario Oficial La Gaceta.

En este breve artículo; en una primera parte, se hará un recorrido por la naturaleza de Costa Rica en cuanto al recurso hídrico, las montañas, el clima y los bosques; y en un segundo apartado, se analizará el tema de las vías comunicación y los medios de transporte. Para esta última sección se realizará un inventario de las obras existentes, para luego, dirigir la mirada hacia las necesidades viales de Costa Rica para 1907.

\section{Una naturaleza pródiga}

La naturaleza fue pródiga para derramar sus dones en este pedacito de América, al situarlo entre los dos grandes océanos de la historia que fueron, son y seguirán siendo las dos inmensas arterias de civilización del globo; cruzó, además sus fértiles campos con ríos caudalosos y en cada montaña hizo brotar diversos manantiales para que las aguas próvidas no faltaran nunca en las tareas de las labranzas (Segarra y Juliá, 1907: p. 460).

Esta descripción corresponde a la abundancia del agua de Costa Rica. Son los ríos y los manantiales la verdadera riqueza del país. Así se hace saber en el caso de los riachuelos que descienden desde las cumbres del Volcán Turrialba:

Deslízase levemente rumorosa, el agua cristalina lamiendo los musgosos pedruscos que á modo de extravagantes anfibios tomara el baño en el centro del cauce: y se mecen á las caricias de la linfa de los retorcidos bejucos que penden de la bóveda ramosa; y temblequean las aplatadas acuáticas, y al frescor de la umbría revuelan impalpables en torno nuestro, mariposas de inverosímil policromía transparente, sutiles hormigones de cuerpo de rubí ceñido por coselete de cristal, y libélulas de oro con alas de diamante... (Segarra y Juliá, 1907: p. 86).

La representación anterior, además de enfatizar la riqueza del recurso hídrico, teje toda una descripción de los ecosistemas asociados a un río de montaña. Continúa con su asombro porque, gracias a la humedad pululante en este sector, se encuentran una gran cantidad de ejemplares como alacranes, víboras, tobobas y exóticas especies vegetales como el maíz gigante cuyos tallos alcanzan a medir tres a cuatro metros de altura.

La importancia de los ríos también se puede evidenciar en la riqueza de las aguas que dan de beber a las llanuras de la región norte, según los autores: "La región llamada á ser en el porvenir que deseamos sea próximo una gran fuente de riqueza para Costa Rica, es la vasta zona que riegan el Río Frío, el San Carlos y el Sarapiquí..." (Segarra y Juliá, 1907: p. 626).

En la región sur también abunda el agua, pero en este caso los visitantes no se esmeran en contemplar su belleza; sino que por el contrario la desmedida cantidad del recurso, a causa de los aguaceros torrenciales, puso en peligro el paso de los 
transeúntes dado que el camino "ofrece, entre otras dificultades, la muy seria de los pasos de las quebradas, peores, que de ordinario á causa de los últimos temporales..." (Segarra y Juliá, 1907: p. 484).

La atracción de las montañas también es reproducida por los viajeros merced al hecho de su escalada hacia las cumbres de los volcanes. Subieron al Irazú y al Turrialba; del primero afirman que durante la noche el frío se asemeja al polar o el europeo, por su altura es totalmente diferente al clima del trópico. (Segarra y Juliá, 1907: p. 414). En tanto que del segundo se refieren como un coloso tranquilo "cuya mole de tres mil metros señala la joroba principal de la cordillera que corta el horizonte por aquel lado" (Segarra y Juliá, 1907: p. 83). Allí la vegetación es "más viril" y el terreno más salvajemente macho; es decir, más agreste e imponente.

Los viajeros también ofrecen una imagen de los diferentes climas del país. Inician con Limón, lugar al que atracan procedentes de la mayor de las Antillas. Sus opiniones no son las más favorables porque ese caserío costero "solo exhibe su amontonamiento de edificios mezquinos, cuyos tejados de zinc se derriten bajo la Iluvia de fuego que cae espesa..." (Segarra y Juliá, 1907: p. 47).

Dadas las condiciones climáticas de Limón se hizo necesaria la inmigración. Para don José y don Pedro::

Hay una razón de peso que explica la supremacía numérica del elemento negro en esta zona excesivamente cálida y poco salubre por lo general: lo relativamente bien que esta gente soporta los rigores del clima que la raza blanca apenas puede soportar... (Segarra y Juliá, 1907: p.66).

El calor excesivo se precipita en el suelo limonense como lluvia de fuego, al igual que sucede en Turrialba y en Guápiles. En este último poblado, los visitantes permanecieron unos días en la Hacienda La Numancia, una vasta propiedad con cobertura boscosa y también dedicada a la ganadería de doble propósito, además de la caballar.

Las temperaturas son un poco menos intensas en el valle de Ujarraz, en uno de sus poblados en Cachí, aunque según los autores de Excursión por América, Costa Rica, es "sofocante el calor de las horas meridianas" (Segarra y Juliá, 1907: p. 446). Allí se encuentra la hacienda propiedad de Mr. Wahle dedicada al cultivo del café

Imagen 3: Hacienda propiedad de Mr. Wahle en Cachí. Fuente: Tomado con fines didácticos de: Segarra, José y Juliá, Joaquín. (1907). Excursión por América, Costa Rica. San José: Imprenta de Avelino Alsina, p. 447.

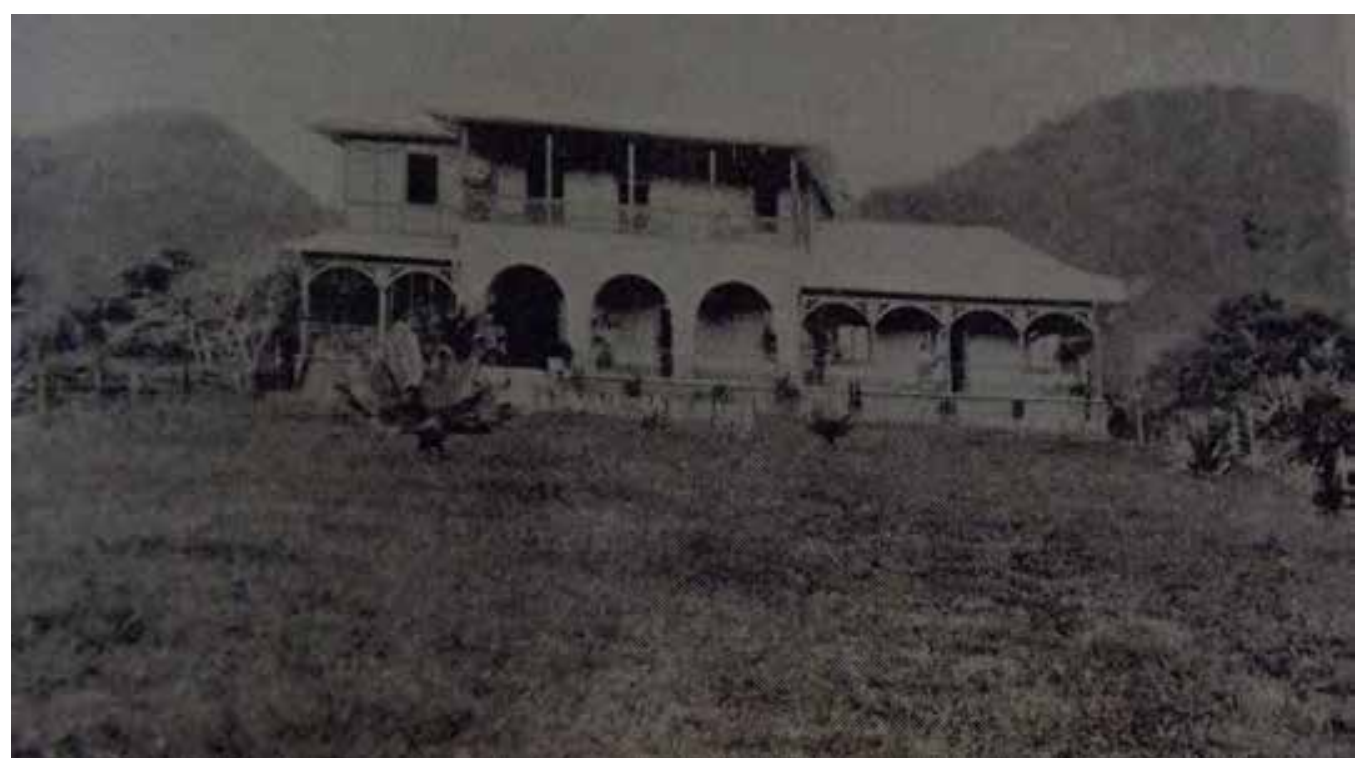


con sombra, una forma de siembra típica de aquellos años anteriores a la revolución verde (Samper, 1993).

Con su visita por Nicoya, una de las poblaciones más antiguas de la comarca, los excursionistas bridan sus impresiones acerca de la calidez del clima, que en este caso es el responsable de la producción de una gran diversidad de frutos. Son "exquisitas frutas que no tienen rival en el país: los mangos, los zapotes, el marañón, el zopilotillo, el mamey, los nísperos, etc." (Segarra y Juliá, 1907: p. 607).

En la misma región del Pacífico, en Orotina -llamada por don José y don Joaquín como Santo Domingo- se distingue por su calor intenso, "deja en nosotros la enervante presión de su calor de horno..." (Segarra y Juliá, 1907: p. 511).

Finalmente, merece un tratamiento aparte el caso de Atenas, que además de ser catalogada como una villa progresista, es vista por los viajeros como excepcional de poseer uno de los mejores climas: "la naturaleza de su suelo rocalloso y la ventajosa altimetría, que la hacen disfrutar [a la villa de Atenas] de un clima muy saludable por lo seco, estando destinada a ser el más prestigioso sanatorio de la República." (En el original aparece esta palabra destacada en cursiva, Segarra y Juliá, 1907: p. 516).

En cuanto a la cubierta forestal::

toda la divina fantasía [...] que florecen en los rosetones y en los arquitrabes de la majestuosa arquitectura de los bosques seculares, y en la cual las ojivas tamizan la luz al través de las vidrieras historiadas [...] forman la sacra corte del sumo sacerdote del rito de la luz y de los colores-el quetzal, cuyo plumaje de púrpura y de tornasol, incienso en al incomparable basílica las aladas irisaciones del oro de los cielos y del zafiro de los mares... (Segarra y Juliá, 1907: p. 483-484).

De lo anterior se desprende que son los bosques el motivo de asombro del visitante. Esta descripción corresponde a la zona sur del país con su diversidad de las especies vegetales, así como su relación con la fauna; y en este caso, se privilegia al ave reinante de la vegetación nubosa: el quetzal. De igual manera, en el mismo sur, pero esta vez en la costa de Dominical "la vegetación es simplemente prodigiosa" (Segarra y Juliá, 1907: p. 472), en un entorno donde la presencia del ser humano es poco visible; esto aunque un vecino de apellido Correa, inmigrante nicaragüense, se dedica al negocio de la extracción de la madera. (Segarra y Juliá, 1907: p. 473).

En tanto, en el Caribe también se alude a la riqueza de la cobertura forestal en los términos siguientes: "anarquía de la naturaleza salvaje que aprisiona aquellos campos de las reglas y preceptos agrícolas entre las lujuriosas rebeldías de bosques inexplorados imponentes en la fragilidad de su vegetación soberbia" (Segarra y Juliá, 1907: p. 53).

En la Península de Nicoya los excursionistas también hacen notar la riqueza forestal, allí se encuentran "abundantes árboles resinosos como copal, el higuerón, el zapote" (Segarra y Juliá, 1907: p. 608) además de los cedros y los caobas. Más adelante se agrega el sentido utilitario del recurso: "He aquí la gran riqueza e inagotable mina para explotar de este privilegiado suelo..." (Segarra y Juliá, 1907: p. 608).

Esta riqueza implica, en términos económicos de mercado, la necesidad de cortar los árboles para la obtención de maderas dedicadas a la carpintería, a la ebanistería y a la construcción en general; esta apreciación de los excursionistas también la hacen extensiva a las llanuras septentrionales de Costa Rica donde el bosque es quien domina y las actividades humanas se hallan enclavadas en medio de la selva, "Las llanuras de San Carlos están cubiertas de bosques que encierran desde las exquisitas maderas de construcción hasta las más ricas y variadas plantas resinosas y tintóreas 
que se ufanan las más fecundas selvas tropicales..." (Segarra y Juliá, 1907: p. 626).

En suma, el interés de don José y de don Joaquín por dedicar parte de su relato a poner énfasis a la riqueza forestal también se dirige, a hacer un llamado de atención para impulsar la explotación maderera; aspecto esencial para comercializar el recurso maderero y también para que en una etapa posterior -una vez derribado el bosque-se pueda introducir el cultivo de productos con un valor comercial. De esta forma, como los señala Anthony Goebel (2012) se produce la inserción "definitiva" de la naturaleza al mercado mundial de Costa Rica.

En la línea anterior, llama la atención el hecho de que el móvil económico de los autores de Excursión por América, Costa Rica incluye también a las tortugas de las cuales se puede aprovechar su concha para la obtención del carey, además de los huevos, la grasa y la carne.

\section{Dotar de vías de comunicación y de medios de transporte}

Las arterias viales son de vital importancia para conectar un país; en el criterio de Segarra y de Juliá es de necesidad imperiosa atender este aspecto medular en la vida de este diminuto Estado. En primer lugar, se aborda el tema de las obras; para en un segundo momento, analizar las pendientes por realizar. En cuanto a la infraestructura ya construida, destaca el Ferrocarril al Atlántico inaugurado con gran pompa en 1890, luego de veinte años de construcción (Stewart, 1991); esta obra permitió la conexión entre el Valle Central y Limón. Su recorrido cruza por una geografía accidentada como lo evidencia el puente sobre el Río Birrís, un afluente del Reventazón. Para don José y don Joaquín, con esta empresa se dio un giro en la economía de una región de Costa Rica, merced al despegue y posterior desarrollo

Imagen 4: Puente

ferroviario sobre el Río Birrís. Fuente: Tomado con fines didácticos de: Segarra, José y Juliá, Joaquín. (1907). Excursión por América, Costa Rica. San José: Imprenta de Avelino Alsina., p. 129.

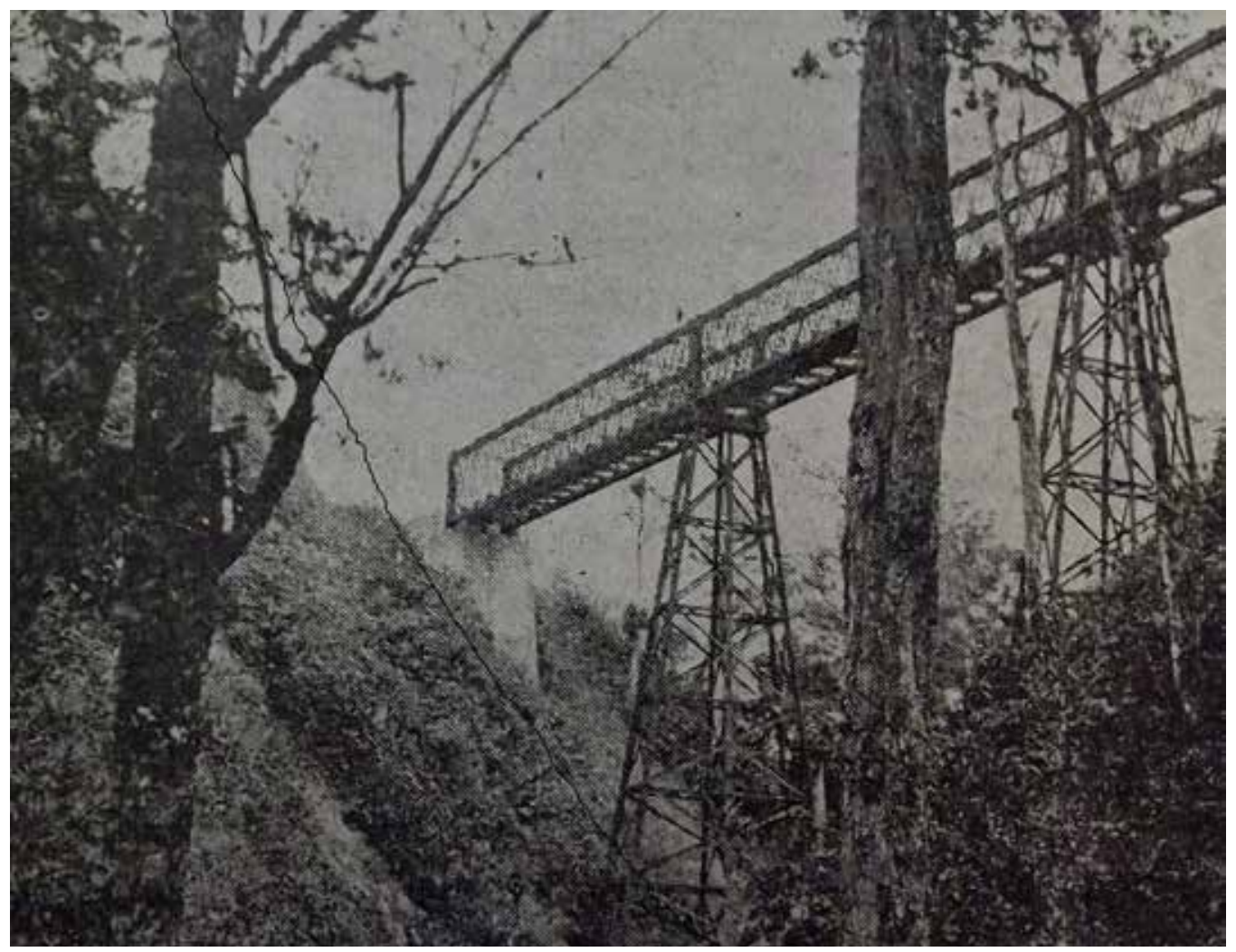


de la plantación bananera:

Hace cuarenta años, el único puerto de Costa Rica en el Atlántico y el más concurrido de al América Central [...] En la construcción del ferrocarril al anterior comenzó a mungir este que hoy es un centro de tráfico de primer orden habiendo determinado el mayor y más efectivo impulso de su desarrollo, la explotación agrícola de que fue y continúa siendo el alma de dicho Mr. Keith: nos referimos al cultivo y exportación de bananos a grande escala. (Segarra y Juliá, 1907: p. 51)

El viaje a bordo del ferrocarril resulta bello por la naturaleza contemplada y admirada por el viajero; el cuadro de diferentes tonos de verdor, exhibe la riqueza natural caribeña. Los excursionistas tomaron el tren, que parte de Limón, a las diez y media de mañana con destino a la capital. A lo largo del recorrido logran también captar las identidades de hierro y humo, como las denomina Carmen Murillo (1995); es el paisaje humano de origen afro apreciado a través de la ventana el vagón, "las fincas á la derecha, haciendas á la izquierda, negras que nos sonríen desde las casas, negras que nos miran desde los campos y bananos por todas partes" (Segarra y Juliá, 1907: p.125).

Además se alude a las actividades económicas propias de las formas de producción doméstica de las familias lugareñas para así obtener el sustento diario. En Turrialba mujeres "de color" ofrecen "con la mayor finura del mundo" a los pasajeros "jugosas ostias de piña" (Segarra y Juliá, 1907: p.127). En tanto, que "así va transcurriendo el viaje; hasta que el tren se mete en poblado, por una calle ancha que determinan dos filas irregulares de casuchas de adobe..." (Segarra y Juliá, 1907: p. 127). Así llegaron a Cartago, la antigua capital colonial construida de bahareque y adobes; es la descripción correspondiente a esa ciudad antes del demoledor terremoto de mayo de 1910,

De pronto,

¿Bizcochos!...

¿Bizcochos!... ¿Confites!...

- ¿Confites!...

¿Bizcochos!... (127)

[...] los bizcochos de Cartago son a modo de pequeñas celosías de masa amarillenta formadas por diminutos "rollitos" (Segarra y Juliá, 1907: p. 128).

De acuerdo con los excursionistas, el Ferrocarril al Atlántico detuvo el desarrollo de Puntarenas; poblado del Pacífico que tuvo que esperar hasta 1897 cuando se inició la obra de construcción de la vía férrea como una obra de tipo estatal (Hall, 1984), que por cierto, era muy bien vista por los ojos de los viajeros por considerarse vital para el desarrollo económico del país. Al respecto:

Perteneciendo éste al Estado, que, naturalmente, no ha de mirar la explotación con el espíritu estrecho de utilitarismo rabioso con que lo miran las empresas particulares, servirá de contrabalance -digamos de freno- a la compañía que ha sabido hacerse dueña del ferrocarril al Atlántico y cuya 
tarifa se fletes encarece considerablemente los productos. (Segarra y Juliá, 1907: p. 528).

En oposición a la Northern, la empresa ferrocarrilera subsidiaria de la UFCo, se piensa en la construcción de una nueva vía -en este caso a Puntarenas- proyecto que levantó las más variadas posiciones de sectores de empresarios y políticos, que externaron su opiniones, muchas veces encontradas acerca de los posibles efectos de un ferrocarril al Pacífico. Ana María Botey (1999), quien destaca este ferrocarril como ente regulador del Estado, comenta que hasta los carreteros, encargados del transporte de abastos y de personas, mostraron su disgusto de cara al desarrollo de este proyecto, esgrimiendo argumentos asociados a la eventual ruina de su actividad en su condición de transportistas. Mientras tanto, don José y don Joaquín muestran su beneplácito para que la obra de conectar el Pacífico logre ser concluida a la mayor brevedad, dadas las ventajas derivadas por la apertura del canal de Panamá al posibilitar acortar el tiempo del transporte del café con destino a los mercados de Europa.

Dentro del recorrido del tramo habilitado del Ferrocarril al Pacífico se destaca su partida desde San José hasta Santo Domingo -antiguo nombre de un distrito de San Mateo, llamado luego Orotina (Noriega, 1923)-; entre los dos puntos geográficos antedichos se tardaban dos horas con treinta minutos. En sí “La línea no tiene nada que envidiar á la del Atlántico en punto á belleza del paisaje, á la importancia de la obra en cuanto á la abrupta naturaleza del terreno que atraviesa; ni tampoco en construcciones de nivelación de defensa y de puentes" (Segarra y Juliá, 1907: p. 510).

Entre los detalles, saltan a la vista los siguientes:

- El puente del Río Grande es "una maravilla de atrevimiento" de la mecánica (Segarra y Juliá, 1907: p. 510).

- La sinuosidad del camino: "un culebreo de un paisaje montuoso sobre toda ponderación" (Segarra y Juliá, 1907: p.510).

- El recorrido por poblados como San Antonio (cabecera lo que iría a ser después el cantón de Belén a partir de 1907); Turrúcares y por supuesto Atenas; esta última "... situada sobre el viejo camino nacional, antes muy frecuentada por se la única vía que comunicaba el interior con el Pacífico - desde Cartago a Puntarenas pasando por Alajuela-..." (Segarra y Juliá, 1907: p. 16).

- Finalmente acerca de la estación final ubicada en Orotina, los excursionistas señalan su importancia porque a partir de este punto se pueden dirigir a: Esparta (Esparza), por su vitalidad económica que se remonta al período colonial, y a Puntarenas, tramo pendiente de colocar la línea férrea, pasando por Coyolar y Pozón; para así entonces concluir exitosamente esta empresa de comunicación.

En cuanto al segundo tema, el de las vías de comunicación por construir, merece mencionarse el del tranvía o ferrocarril de vía angosta entre Alajuela y San Ramón, vía Grecia, que conectaría las diferentes comunidades del Occidente del Valle Central. Esta área se había empezado al poblar a la luz del apogeo de la caficultura a partir de la década de 1840 (Badilla y Solórzano, 2010). El tranvía era el objetivo vial de un proyecto de ley propuesto por el diputado Juan Vega Lizano (Segarra y Juliá, 1907: p. 520). A su vez Segarra y Juliá observan a San Ramón, el destino final de esta obra de comunicación como "una simpática ciudad, una futura capital de una 


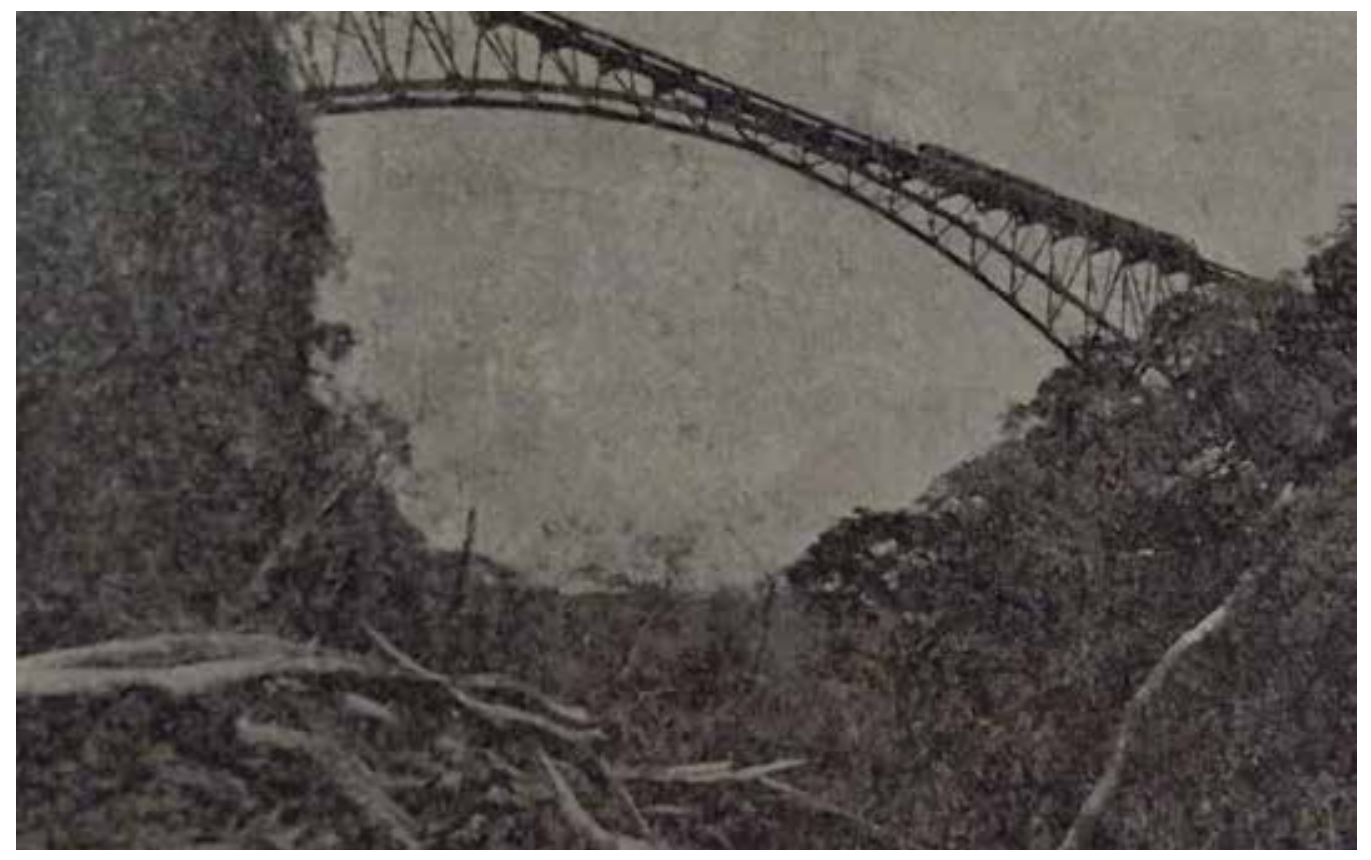

Imagen 5: Puente

ferroviario sobre el Río Grande. Fuente:

Tomado con fines didácticos de: Segarra, José y Juliá, Joaquín. (1907). Excursión por América, Costa Rica. San José: Imprenta de Avelino Alsina, p. 511.

nueva provincia -situada en una hermosa altiplanicie al O. del Río Grande y al E. de la línea divisoria que separa la cuenca de este río de la del Barranca..." (Segarra y Juliá, 1907: p. 520).

Sin duda, tenían razón los visitantes españoles al asignarle ese lugar preponderante a San Ramón, pues como bien lo documentan Yamileth González y María Pérez (1993), este poblado occidental llegó a levantar los deseos de sus habitantes en aras de conformar una provincia independiente de Alajuela, unos siete años después de que se publicara la obra de don José y de don Joaquín.

El otro ferrocarril por construir es el que conectaría las llanuras del norte, región de un amplio potencial natural y productivo, sobre todo por el caucho, el cacao y los pastos. Para los autores, quienes se vieron imposibilitados de visitar esas tierras, "es inminente la construcción de un ferrocarril que habrá de determinar forzosamente un incalculable venero de prosperidad para el país..." (Segarra y Juliá, 1907: p. 626).

Por último, en la región sur de Costa Rica los excursionistas son incisivos y ponen en relieve la impostergable misión de conectar importantes áreas. La primera vía debía ser construida como alterna a la del Cerro del Muerte; así se podría llegar a El General con mayor facilidad. Esta localidad está en un momento importante del proceso de colonización agrícola y no es justo que por la ruta del cerro se derroche tiempo, dadas las condiciones agrestes de la montaña. En palabras de los viajeros: “El Valle de El General reúne condiciones inmejorables - por el clima, por su salubridad y por sus buenas tierras- para atraer a él una seria corriente de inmigración nacional y extranjera" (Segarra y Juliá, 1907: p. 501).

Se debe por tanto buscar una salida hacia la costa para así sacar la producción agropecuaria. De esta manera, ser pretende integrar también a los poblados de Dominical y de Buenos Aires, este último "homónimo de la opulenta metrópoli del Plata en su pequeño poblado que cada día aumenta en importancia, y está destinado á ser, a su vez, la metrópoli de esta rica cuanto abanderada región del Sur" (Segarra y Juliá, 1907: p. 486).

Este caserío meridional cuenta para la época con aproximadamente 700 habitantes distribuidos en un centenar de ranchos. Según los visitantes, hacia 1895 si acaso residían en Buenos Aires unas cien almas. Por esto urge que el gobierno asuma con 
diligencia y celeridad las obras correspondientes a fin de brindar las vías de comunicación necesarias para el despegue económico de Costa Rica.

Como punto y final del tema de los caminos, los excursionistas no dudan en brindar un espaldarazo al proyecto planteado por la Dirección de Obras Públicas que consistía en la implantación de la implantación de un "servicio de peones camineros", a la usanza europea, con la finalidad de conservar y reparar las carreteras existentes (Segarra y Juliá, 1907: p. 519).

\section{Reflexiones finales}

El discurso oficial tan en boga en la actualidad que postula a Costa Rica como un país verde, cien por ciento natural, puede probablemente encontrar sus orígenes y posterior evolución en los textos de los viajeros. En el texto escrito por Segarra y Juliá se evidencia que ha habido históricamente un interés en enfatizar el potencial inagotable de los recursos naturales: el verdor sempiterno de los pastos, la abundancia del recurso hídrico y la riqueza forestal del país, aunada desde luego, a la posibilidad de explotar las tierras feraces con la siembra de cultivos para su comercio respectivo. Sin embargo, al otro lado de la moneda, la realidad de Costa Rica es vista por los excursionistas como un país que pese a sus múltiples encantos naturales merece la realizar ingentes esfuerzos en al construcción de infraestructura vial.

Así las cosas, los encantos de Costa Rica como país enclavado en el istmo centroamericano se tienden a desvanecer en cuanto se colocan frente al déficit de caminos y medio de transporte. Hacía falta de concluir un trecho nada despreciable del Ferrocarril el Pacífico, que iba desde Orotina hasta Puntarenas; era necesario unir el Occidente del Valle Central mediante un tranvía que partiera de la ciudad de Alajuela con destino a San Ramón; de igual manera, era de interés establecer una vía de comunicación que diera vida a la zona norte y que además fuera capaz de unir las llanura del Sarapiquí y Río Frío. Y por último, era menester atender las necesidades de dotar de una salida al Pacífico para así buscar una ruta alterna al Valle de El General.

El propósito de Segarra y de Juliá era insistir ante las autoridades acerca de la necesidad de dotar de rutas y de vías de comunicación que fueran a su vez portadores del progreso acorde con el ideario liberal de entonces. La construcción de caminos y de ferrocarriles, derivaría en el aumento de los rendimientos agrícolas en virtud de la ruralidad del país. En caso de ser posible la mejora vial, entonces se ofrecerían condiciones atractivas para la inmigración de nacionales y de extranjeros interesados en explotar los recursos naturales tan preciados como las maderas; todo esto a su vez constituye uno de los pilares de la noción de crecimiento de la época.

Si se tuviera que traer los planteamientos de Segarra y de Juliá a la actualidad más cercana, se podría anotar, en primer lugar, que el interés de exportar la idea de una país con una exuberante riqueza natural -hoy denominada biodiversidad-todavía constituye uno de los bastiones sobre los que descansa la marca país de Costa Rica vendida en el extranjero, noción acuñada en los tiempos del mercadeo y de la globalización. Queda por verse, sin embargo, qué tan verdes son tanto las prácticas productivas como los habitantes de este país.

Se podría también afirmar, sin ambages, que la preocupación de los autores referente a las vías de comunicación es también contemporánea a la de cualquier ciudadano que habite la Costa Rica de hoy. El Ferrocarril al Atlántico, concluido en 1890 como un monumento al progreso financiado por el capital privado, feneció en 1995 junto con su homólogo del Pacífico, construido con finanzas públicas. Tampoco se construyó el tranvía entre Alajuela y San Ramón.

A ciento diez años después de la visita de Segarra y Juliá, Costa Rica se trata de 
vender como un destino verde para los turistas, eso sí con una cobertura forestal nativa correspondiente solo a las áreas protegidas mediante reservas y parques nacionales. Y como si no fuera suficiente, el país se encuentra con un rezago crónico de décadas, o tal vez de un siglo, en materia de vías de comunicación.

\section{Bibliografía}

\section{FUENTE PRIMARIA PUBLICADA:}

Segarra, José y Juliá, Joaquín. (1907). Excursión por América, Costa Rica. San José: Imprenta de Avelino Alsina.

\section{TESIS:}

Goebel, Anthony. (2012). La explotación económica de los bosques y su rol en la inserción "definitiva" de la naturaleza en el mercado mundial contemporáneo: el caso de Costa Rica (1883-1955). Tesis de doctorado en Historia, Sistema de Estudios de Posgrado. Universidad de Costa Rica.

\section{REVISTAS:}

Botey, Ana Marìa. (1999). "El Ferrocarril al Pacífico: un ente de regulación en crisis permanente (1880-1972)". Anuarios de Estudios Centroamericanos. 25 (1): pp. 139-157.

González, Yamileth y Pérez, María. (1993). “Iglesia y poder político: un fragmento de la historia comunal, 1866-1920". Revista de Ciencias Sociales. 61 (1): 97-113.

\section{LIBROS:}

Badilla, Maynor y Solórzano, William. (2010). De territorio a región: Bases estructurales para la creación de las regiones de Occidente y Norte de Costa Rica. 18211955. San José: Sociedad Editora Alquimia 2000.

Clark, Patricia, Goebel, Anthony y Rivero, Francesca. (2014). "Historiografía de la historia ambiental". En: Díaz, David; Molina, Iván y Viales, Ronny. (ed.). Historiografía costarricense en la primera década del siglo XXI: tendencias, avances e innovaciones. San José: EUCR.

Hall, Carolyn. (1984). Costa Rica: una interpretación geográfica con perspectiva histórica. San José: ECR.

Molina, Iván. (2003). “Dana Gardner Munro: del olvido a la recuperación intelectual de un imperialista democrático". Munro Dana, Las cinco repúblicas de Centroamérica: desarrollo político y económico y relaciones con Estados Unidos. San José: EUCR.

Murillo, Carmen. (1995). Identidades de Hierro y Humo: La construcción del Ferrocarril al Atlántico 1870-1890. San José: Porvenir.

Noriega, Félix. (1923). Diccionario geográfico de Costa Rica. San José: Imprenta Nacional.

Samper, Mario. (1994). "Café, trabajo y sociedad en Centroamérica, (1870-1930): una historia común y divergente". Víctor Hugo Acuña (edit). Historia General de Centroamérica. FLACSO-Sociedad Estatal Quinto Centenario: Madrid.

Steward, Watt. (1991). Keith y Costa Rica. San José: ECR. 
